\title{
Synovial sarcoma arising in the vulva cytogenetically confirmed by SYT break-apart rearrangement fluorescence in situ hybridization: A case report and discussion of diagnostic methods
}

\author{
SHIGETO KAWAUCHI ${ }^{1}$, KOICHIRO IHARA ${ }^{2}$, KEI NISHIKAWA ${ }^{1}$, NORIHIRO SUGINO ${ }^{3}$, \\ MUTSUO TAKAHASHI $^{4}$ and KOHSUKE SASAKI ${ }^{1}$ \\ Departments of ${ }^{1}$ Pathology, ${ }^{2}$ Orthopedic Surgery, ${ }^{3}$ Gynecology, and ${ }^{4}$ Faculty of Health Sciences, \\ Yamaguchi University School of Medicine, Yamaguchi 755-8505, Japan
}

Received February 14, 2012; Accepted June 8, 2012

DOI: $10.3892 / \mathrm{ol} .2012 .857$

\begin{abstract}
Synovial sarcoma (SS) is a soft tissue sarcoma of unknown histogenesis that rarely occurs in the female genital tract. We report a case of SS occurring in the right vulva of a young Japanese female. The tumor was composed of poorly differentiated rounded cell areas, surrounded by fibroblastic spindle-shaped cell areas. Immunohistochemically, the tumor cells were focally positive for cytokeratin, vimentin, CD99, Bcl-2 and neuron-specific enolase. The tumor was suspected, but was difficult to confirm as it was an SS based solely on lightmicroscopic and immunohistochemical findings. Although reverse transcription polymerase chain reaction (RT-PCR) failed to detect SS-specific SYT-SSX fusion gene transcripts using an RNA sample extracted from the formalin-fixed paraffin-embedded tumor tissue, SYT break-apart rearrangement fluorescence in situ hybridization (SYT bar-FISH) successfully confirmed our diagnosis of SS for the tumor. Thus, SYT bar-FISH may be more suitable for the purpose of the molecular diagnosis of SS than conventional RT-PCR when using archival formalin-fixed paraffin-embedded tissue specimens.
\end{abstract}

\section{Introduction}

Synovial sarcoma (SS) is a soft tissue sarcoma of unknown histogenesis and comprises approximately $10 \%$ of all soft tissue sarcomas, occurring most frequently in adolescents and young adults $(1,2)$. Morphologically, SSs are classified into three types, i.e., the classical biphasic type that is composed of both epithelial and spindled-cell components, the monophasic

Correspondence to: Dr Shigeto Kawauchi, Department of Pathology, Yamaguchi University School of Medicine, Minamikogushi, Ube, Yamaguchi 755-8505, Japan

E-mail:shig@yamaguchi-u.ac.jp

Key words: synovial sarcoma, vulva, differential diagnosis, fluorescence in situ hybridization, reverse transcription-polymerase chain reaction type composed of either epithelial or spindle-cell components, and the poorly differentiated type composed of small round cells (1).

A diagnosis of SS is relatively straightforward for the classical biphasic type, however, it may be challenging for the monophasic fibrous and poorly differentiated types. This is partially due to the fact that, although SS usually shows a dual mesenchymal and epithelial differentiation light-microscopically and immunohistochemically, the differentiation may be ambiguous in the monophasic fibrous and poorly differentiated types $(1,2)$. Furthermore, although SS typically arises in the deep soft tissue of the extremities, especially in the thigh and knee joints, rare cases have been described in a variety of anatomic sites and organs, including the female genital tract $(3,4)$. However, SS of the female genital tract is extremely rare, potentially making a diagnosis of SS difficult when this is the primary site.

Tumor-specific chromosomal translocations and associated fusion genes have attracted great interest due to their possible roles as diagnostic markers and prognostic predictors in soft tissue sarcomas (5). Over $95 \%$ of SSs are characterized by a reciprocal chromosomal translocation $\mathrm{t}(\mathrm{X} ; 18)(\mathrm{p} 11.2 ; \mathrm{q} 11.2)$, which is not detectable as a non-random chromosomal translocation in any sarcomas, with the exception of SS. The translocation usually fuses SYT onto chromosome $18 \mathrm{q}$ with either SSX1 or SSX2 on chromosome Xp (5). Furthermore, a rare SYT-SSX4 fusion was also reported as an SS-specific chimeric gene (6). Currently, the detection of these fusion genes is considered to have full diagnostic validity for diagnosing SS. Although reverse transcription-polymerase chain reaction (RT-PCR) is the most popular method used to detect SS-specific fusion gene transcripts and to confirm a diagnosis of SS, SYT break-apart rearrangement fluorescence in situ hybridization (SYT bar-FISH) has recently been introduced as an alternative molecular method to diagnose SS (7-9).

We report a case of SS occurring in the right vulva of a young Japanese female. Based solely on light-microscopic and immunohistochemical findings, the tumor was difficult to diagnose as SS. Although RT-PCR failed to detect SS-specific SYT-SSX fusion gene transcripts using sample RNA extracted from the formalin-fixed, paraffin-embedded tumor tissue, SYT 
bar-FISH successfully confirmed the diagnosis of SS. In the present study, we also discuss issues concerning the diagnosis of SS, along with the methodological considerations regarding molecular diagnosis.

A 21-year-old Japanese female, nulligravida and nullipara, was referred to the Yamaguchi University Hospital with a complaint of a palpable right vulvar mass. The mass was elastic hard in consistency, slightly tender, and smaller than a fist. A computed tomography examination demonstrated a relatively well-demarcated solid tumor of approximately 9x8x7 cm in size with central cysts. A needle biopsy examination demonstrated solid nests of poorly differentiated small round cells with distinct vasculature. Based on a putative diagnosis of soft tissue sarcoma, tumor resection was performed. Twenty-eight months following surgery, the tumor recurred in the right lung, and four years following the first surgery, the patient succumbed to multiple recurrence of the tumor.

The present study was approved by the ethics commettee of Yamaguchi University School of Medicine, Yamaguchi, Japan. Informed consent was obtained from the patient.

\section{Materials and methods}

Preparation of tissues. The resected tumor tissues were forwarded for overnight fixation in $10 \%$ neutral-buffered formalin at room temperature (RT), routinely processed, and embedded in paraffin-wax. Tumor tissue sections $(4 \mu \mathrm{m})$ were cut from the paraffin-embedded tumor tissue blocks and prepared for hematoxylin and eosin (H\&E) staining, immunohistochemistry and SYT bar-FISH analysis. For RT-PCR, multiple $10-\mu \mathrm{m}$-thick tumor tissue sections were cut from the paraffin-embedded tumor tissue blocks.

Immunohistochemistry. For antigen retrieval, deparaffinized and rehydrated sample tissue sections were pretreated by microwave irradiation for 20-30 $\mathrm{min}$ in $0.01 \mathrm{~mol} / 1$ citratebuffered saline (pH 6.0). Endogenous peroxidase activity was blocked by incubation with $0.3 \% \mathrm{H}_{2} \mathrm{O}_{2}$ solution for $30 \mathrm{~min}$. The tissue sections were then incubated with mouse monoclonal antibodies against cytokeratin (clone AE1/AE3, diluted at 1:100, Dako, Copenhagen, Denmark), CD56 (clone 1B6, Nichirei, Tokyo, Japan), neuron-specific enolase (NSE) (clone BBS/NC/VI-H14, diluted at 1:100, Dako), vimentin (clone V9, diluted at 1:25, Dako), CD34 (clone NU-4A1, diluted at 1:50, Nichirei), CD99 (clone 12E7, diluted at 1:50, Dako), Bcl-2 (clone 124, diluted at 1:100, Dako), muscle-specific actin (clone HHF35, diluted at 1:50, Dako) and desmin (clone D33, diluted at 1:100, Dako), or a rabbit polyclonal antibody against S100 protein (S100, diluted at 1:1000, Dako) at $4^{\circ} \mathrm{C}$ overnight. Subsequent reactions were performed by the streptavidin-biotin complex/horseradish peroxidase method using a Histofine SAB-PO (M) or (R) immunohistochemical staining kit (Nichirei, Tokyo, Japan) according to the manufacturer's instructions.

Break-apart rearrangement FISH and RT-PCR. SYT barFISH was carried out using a LSI SYT (18q11.2) Dual Color, Break-Apart Rearrangement Probe kit (Vysis, Downers Grove, IL, USA) according to the manufacturer's instructions, with minor modifications. A tumor tissue section (4- $\mu \mathrm{m})$ placed on a glass slide was dewaxed, rehydrated, incubated in a $0.05 \%$ pepsin $/ 0.1 \mathrm{~mol} / 1 \mathrm{HCl}$ solution at $37^{\circ} \mathrm{C}$ for $10 \mathrm{~min}$, and washed twice in phosphate-buffered saline, $\mathrm{pH} 7.4$, at RT for $5 \mathrm{~min}$. The section was subjected to microwave pretreatment with $0.1 \mathrm{~mol} / 1$ sodium citrate buffer, $\mathrm{pH}$ 6.0, dehydrated in a graded series of ethanol solutions, incubated in acetone at $-20^{\circ} \mathrm{C}$ for $10 \mathrm{~min}$, and fixed in Carnoy's solution at RT for $5 \mathrm{~min}$. The section was denatured in $70 \%$ formamide/2X standard sodium citrate at $73^{\circ} \mathrm{C}$ for $5 \mathrm{~min}$. A $10 \mu \mathrm{l}$ aliquot of the SYT bar-FISH probe (5'-SYT SO/3'-SYT SG, Vysis) mixture was also denatured at $73^{\circ} \mathrm{C}$ for $5 \mathrm{~min}$ and applied to the denatured sample tissue section. The section was then covered with a cover glass, sealed with rubber cement, and incubated at $37^{\circ} \mathrm{C}$ for $72 \mathrm{~h}$ in a humidified chamber. Post-hybridization washes were routinely performed and the section was counterstained with a DAPI-II antifade solution (Vysis).

The section was observed for FISH signals on each tumor cell nucleus under a BX60 fluorescence microscope (Olympus, Tokyo, Japan). In the enumeration of FISH signals, overlapping cells and cells with indistinct fluorescence signals were excluded. The number of tumor cell nuclei with three fluorescence signals, i.e., the SpectrumOrange-SpectrumGreen overlapping yellowish signals, the SpectrumOrange 5'-SYT signal and the SpectrumGreen 3'-SYT signal, were calculated per over 100 tumor cell nuclei for the section. When the ratio of the number of tumor cell nuclei carrying one overlapped and two separate fluorescent signals for the total number of tumor cell nuclei counted was $>0.7$, the tumor was considered to have a SYT gene break, being diagnostic of SS.

Furthermore, RT-PCR for SYT-SSX1 and SYT-SSX2 fusion gene transcripts were carried out according to the method described in a previous study (10). Appropriate positive and negative controls were included in the RT-PCR examination.

\section{Results}

Gross findings and histopathology. On the cut surface, the tumor was elastic hard to moderately soft in consistency and brownish to tan in color, accompanied by some areas of hemorrhaging and 0.5 - to $2 \mathrm{~cm}$-sized cystic spaces filled with brownish fluid.

Microscopically, the tumor showed a relatively welldemarcated nodular region composed of small rounded cells with distinct vasculature, surrounded by a spindle-cell area composed of fibroblastic cells, accompanied by cellular and myxoedematous portions (Fig. 1A). The former region was composed of a diffuse proliferation of small-rounded tumor cells with distinct staghorn blood vessels (Fig. 1B). The rounded tumor cells were characterized by round to oval nuclei, occasional conspicuous nucleoli, and scant eosinophilic to pale cytoplasm. The latter region demonstrated cellular fascicles of spindle-shaped fibroblastic tumor cells intermingled with collagen bundles, focal hyalinization, and geographic myxoid degeneration (Fig. 1C). The spindled tumor cells were characterized by plump-spindled nuclei, indistinct nucleoli, and eosinophilic to pale cytoplasm (Fig. 1D). Herringbone (Fig. 1E) and nuclear palisading (Fig. 1F) arrangements of the tumor cells were focally observed. 

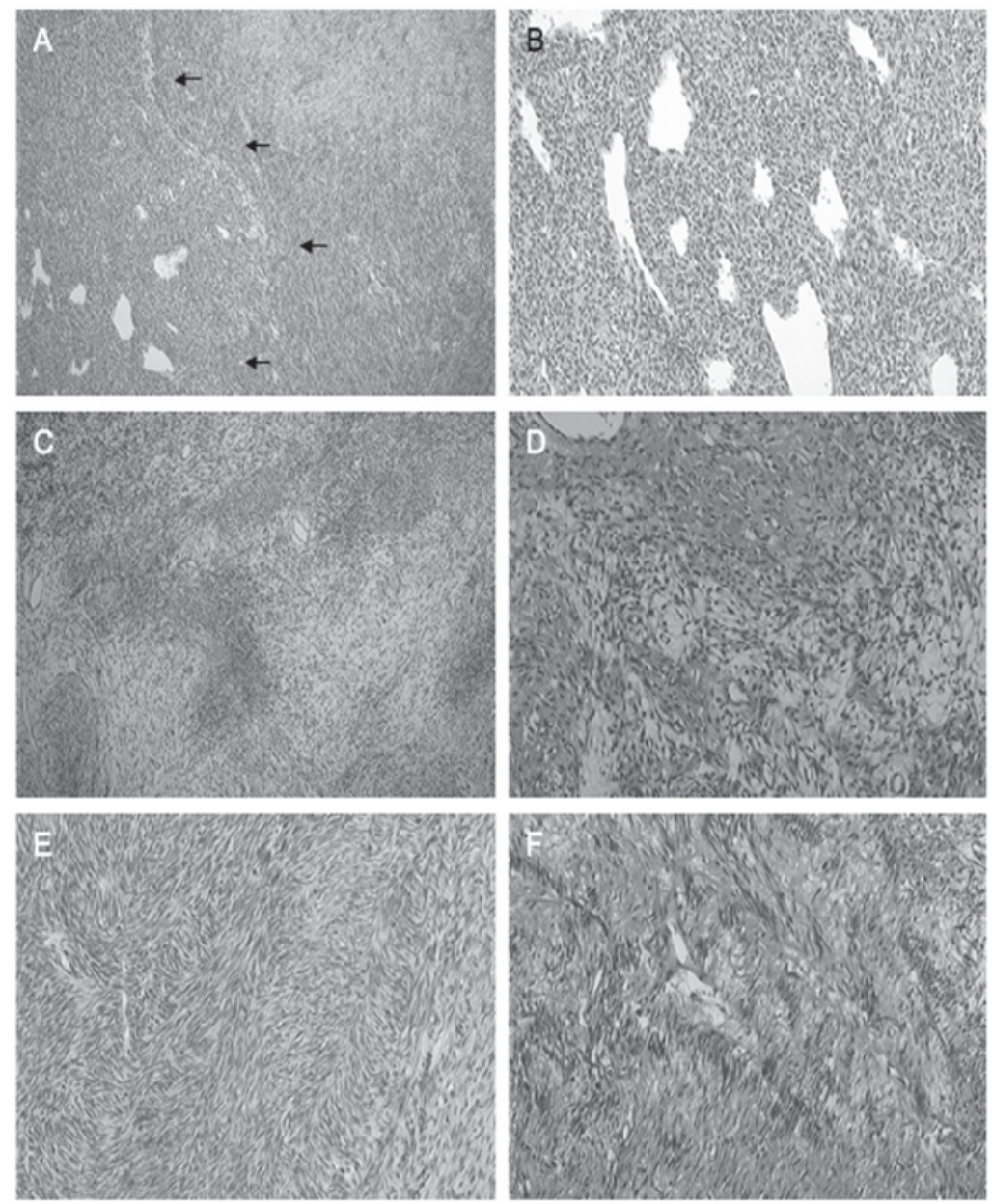

Figure 1. The microscopic findings of the vulvar tumor. (A) The tumor shows a well-demarcated nodular region composed of rounded cells (right) and a surrounding spindle-cell region composed of fibroblastic spindle-shaped cells (left). The two regions are relatively well demarcated (small arrows). (B) The former region demonstrating a diffuse proliferation of small-round tumor cells with distinct staghorn-shaped blood vessels. (C) The latter region showing fascicles of spindle-shaped fibroblastic cells with hyalinized collagen bundles and myxoid degeneration. (D) The spindle-shaped tumor cells are characterized by plump-spindled nuclei, indistinct nucleoli, and eosinophilic to pale cytoplasm. (E) and (F) The herringbone (E) and nuclear palisading (F) arrangements of the tumor cells were focally observed in the tumor.

Immunohistochemistry. Immunohistochemically, the tumor cells were diffusely positive for vimentin (Fig. 2A), S100 (Fig. 2B), Bcl-2 (Fig. 2C), NSE (Fig. 2D) and CD56 in both the spindle-cell and round-cell components, and were focally positive for cytokeratin (Fig. 2E) and CD99 (Fig. 2F) in the round-cell region. The tumor cells were negative for CD34 and muscle-specific actin in both the spindle-cell and round-cell regions.

FISH and RT-PCR. The LSI SYT bar-FISH probe consists of a mixture of two FISH DNA probes. The first probe labeled with SpectrumOrange extends distally from the SYT gene. The second probe labeled with SpectrumGreen lies 3'- or proximal to the SYT gene. In normal metaphase cells without the SYT gene break, the two fusion orange signals are observed. However, in abnormal cells carrying a SYT gene break, the cells usually demonstrate one fusion orange, one red and one green signal pattern (Fig. 3A). This makes it easy to differentiate the sarcoma cells from inflammatory and non-neoplastic stromal cells under fluorescence microscopy. In the present study, over $70 \%$ of the tumor cell nuclei carried one red, one green, and one fusion orange signal in the tumor cells of both the poorly differentiated areas and monophasic fibrous areas (Fig. 3B). A small proportion of the tumor cells had a reduced number of FISH signals, probably due to the nuclear truncation that occurred during the preparation of the tissue sections.

The RT-PCR examination failed to detect the SS-specific SYT-SSX fusion gene transcripts specific for SS, probably due to the poor quality of the RNA samples used in the RT-PCR examination from the formalin-fixed, paraffin-embedded sarcoma tissue (data not shown).

\section{Discussion}

SS rarely arises in the female genital tract. To the best of our knowledge, only 13 SSs of the female genital tract have been reported previously, including 8 tumors in the vulva $(3,4), 3$ in the vagina $(4,11)$, and one each in the fallopian tube (12) and the ovary (13). Recently, various techniques have been used to detect a $\mathrm{t}(\mathrm{X} ; 18)(\mathrm{p} 11.2 ; \mathrm{q} 11.2)$ translocation or SYT-SSX fusion gene transcripts as a molecular marker to confirm a diagnosis of SS. These techniques include short-term tumor cell culture and karyotyping, RT-PCR for SS-specific SYT-SSX fusion gene transcripts, and SYT bar-FISH. Among these methods, 

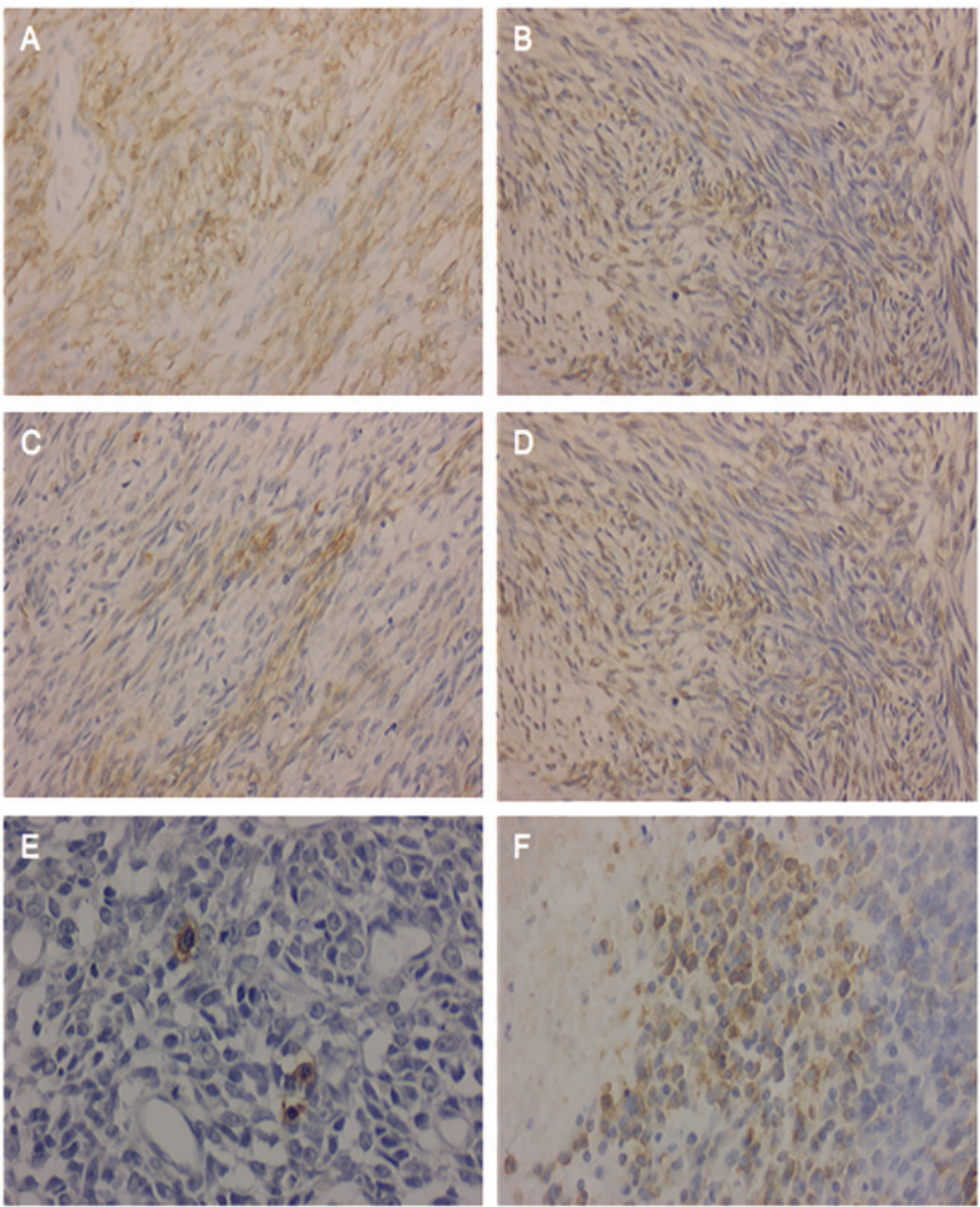

Figure 2. The immunohistochemical findings of the vulvar tumor. (A) to (D), the tumor cells are positive for (A) vimentin, (B) S100 protein, (C) Bcl-2 and (D) neuron-specific enolase (NSE) in the spindle-cell and round-cell regions. (E) and (F), the tumor cells are focally positive for (E) cytokeratin and (F) CD99 in the round-cell region.
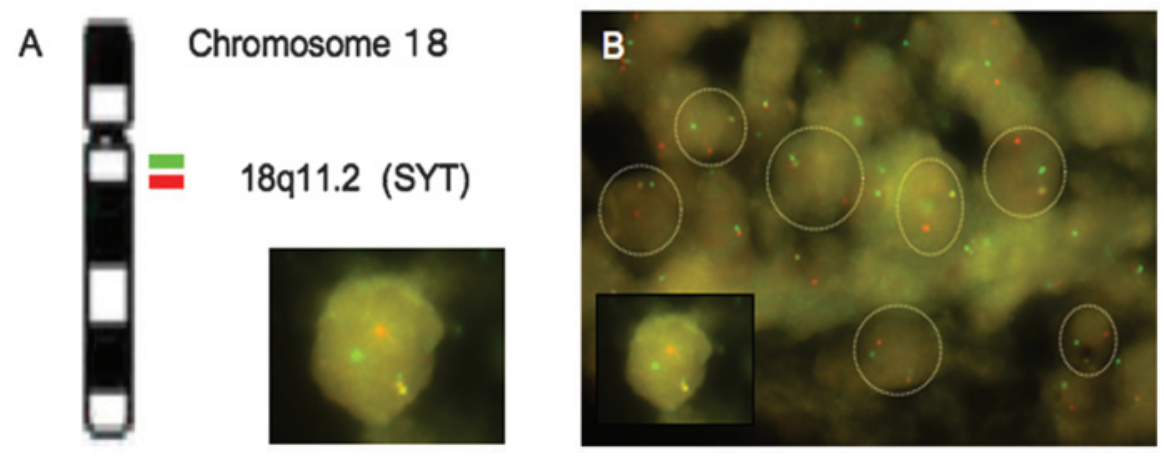

Figure 3. (A) The LSI SYT break-apart rearrangement fluorescence in situ hybridization (FISH) probe consists of a mixture of two FISH DNA probes. The first probe labeled with SpectrumOrange extends distally from the SYT gene (red line). The second probe labeled with SpectrumGreen lies 3'- or proximal to the SYT gene (green line). Cells carrying the SYT gene break specific for synovial sarcoma usually demonstrate one red, one green, and one fusion orange signal pattern. (B) Over $70 \%$ of the tumor cell nuclei exhibit the one red, one green, and one fusion orange signal pattern.

RT-PCR is the most popular, and appears to be regarded as a standard method for diagnosing SS (4). Among the 13 SSs arising in the female genital tract, eight were monophasic fibrous or poorly differentiated SSs and were diagnostically confirmed by RT-PCR to have the SYT-SSX fusion gene transcripts $(3,4,11-13)$.
The present tumor was difficult to diagnose based solely on the light-microscopic and immunohistochemical findings. Microscopically, the tumor was composed of well-demarcated, spindled-cell areas and poorly differentiated small round cell areas. The former showed cellular fascicles of spindleshaped cells, focally exhibiting a herringbone pattern and a 
nuclear palisading arrangement of the tumor cells with focal myxoedematous to collagenous portions. The latter were characterized by distinct vasculature with a staghorn configuration. Furthermore, the tumor cells were, immunohistochemically at least, focally positive for cytokeratin, vimentin, CD99, Bcl-2, and NSE $(1,2)$.

Taking these findings into consideration, the major differential diagnoses of the present tumor were considered to be fibrosarcoma, malignant peripheral nerve sheath tumor (MPNST), myopericytoma, solitary fibrous tumor (SFT) and SS. Although regions mimicking fibrosarcoma and myopericytoma were present in the tumor, they were observed only sporadically. Therefore, these diagnoses could be excluded SFT and MPNST were more problematic differential diagnoses. The present tumor was immunohistochemically negative for CD34 and there were no histories of neurofibromatosis in the patient or her family. Therefore, we eventually excluded SFT and MPNST when making a differential diagnosis.

Although SS was selected as the putative diagnosis of the present tumor from the differential diagnoses presented, evidence supporting diagnostic confirmation was required. For this purpose, RT-PCR for the SYT-SSX fusion gene transcripts was performed. Since there were no fresh-frozen tumor tissues available for the RT-PCR examination, the archival formalin-fixed, paraffin-embedded tumor tissue blocks were used to extract RNA. However, it was difficult to retrieve a sufficient quality and quantity of sample RNA suitable for RT-PCR from the paraffin-embedded tissues. Although RT-PCR is a sensitive method that may be used to detect target RNA from a small amount of sample RNA, it is sometimes difficult to obtain RNA samples suitable for RT-PCR from archival formalin-fixed paraffin-embedded tissues $(8,14)$. This is partially due to the fact that RNA tends to be degraded by prolonged formalin fixation and/or ubiquitously present RNases when there is inappropriate tissue handling. Such inappropriate RNA samples may generate a false-negative or even false-positive result in a RT-PCR analysis.

Since FISH may be more easily and reliably used, even with an archival formalin-fixed, paraffin-embedded sample compared to RT-PCR $(8,9)$, SYT bar-FISH may be more suitable for a diagnosis of SS than RT-PCR. There have been few studies dealing with the methodological comparison between RT-PCR and SYT bar-FISH for the diagnosis of SS based on a considerable number of SS cases. For example, Amary et al reported that a combination of molecular methods, including RT-PCR and SYT bar-FISH, had a 96\% sensitivity and $100 \%$ specificity (8). Sun et al compared the diagnostic efficiency of SYT bar-FISH and RT-PCR in diagnosing 255 cases of SS, and concluded that the efficiency of FISH was comparable to or even higher than that of RT-PCR (9).

In the present case, unlike RT-PCR, SYT bar-FISH successfully confirmed the diagnosis of SS by detecting a pair of split SYT signals for the present tumor. In a typical pathology laboratory unfamiliar with the potential issues regarding RNA experiments, SYT bar-FISH may be more suitable for detecting a tumor-specific chromosomal translocation and diagnosing difficult cases of SS. Furthermore, in the current tumor, a pair of split SYT signals was exclusively observed in the tumor cells of the monophasic fibrous and poorly differentiated components, supporting the hypothesis that these components are derived from the same tumor stem cell in spite of their morphologic differences.

In conclusion, we have described a diagnostically difficult case of vulvar SS that was composed of monophasic fibrous and poorly differentiated components. In the present case of SS, SYT bar-FISH, but not RT-PCR, was used to successfully validate our diagnosis of SS. For the diagnosis of SS arising in unusual anatomic sites, frozen tumor tissue samples may be difficult to prepare for the molecular diagnosis. In such a situation, FISH may be more suitable for the detection of the SS-specific chromosomal translocation and the diagnosis of SS using archival formalin-fixed, paraffin-embedded tumor tissue specimens than RT-PCR.

\section{References}

1. Weiss SW and Goldblum JR: Malignant soft tissue tumors of uncertain type. In: Weiss SW and Goldblum JR (eds). Enzinger and Weiss's Soft Tissue Tumors. Mosby, St. Louis, pp1483-1571, 2001.

2. Fisher C, de Bruijin DHR and Geurts van Kessel A: Synovial sarcoma. In: Fletcher CDM, Unni KK, Mertens F (eds). World Health Organization Classification of Tumours. Pathology and Genetics. Tumours of Soft Tissue and Bone. IARC Press, Lyon, pp200-204, 2002.

3. Ambani DS, White B, Kaplan AL and Alberto A: A case of monophasic synovial sarcoma presenting as a vulvar mass. Gynecol Oncol 100: 433-436, 2006.

4. Sumathi VP, Fisher C, Williams A, Meis JM, Ganesan R, Kindblom LG and McCluggage WG: Synovial sarcoma of the vulva and vagina: a clinicopathologic and molecular genetic study of 4 cases. Int J Gynecol Pathol 30: 84-91, 2010.

5. Melzer ES: Molecular genetics of soft tissue tumors. In: Weiss SW and Goldblum JR (eds). Enzinger and Weiss's Soft Tissue Tumors. Mosby, St. Louis, pp125-146, 2001.

6. Skytting B, Nilsson G, Brodin B, Xie Y, Lundeberg J, Uhlén M and Larsson O: A novel fusion gene, SYT-SSX4, in synovial sarcoma. J Natl Cancer Inst 91: 974-975, 1999.

7. Terry J, Barry TS, Horsman DE, Hsu FD, Gown AM, Huntsman DG and Nielsen TO: Fluorescence in situ hybridization for the detection of $t(X ; 18)(p 11.2 ; q 11.2)$ in a synovial sarcoma tissue microarray using a breakapart-style probe. Diagn Mol Pathol 14: 77-82, 2005.

8. Amary MF, Berisha F, Bernardi Fdel C, Herbert A, James M, Reis-Filho JS, Fisher C, Nicholson AG, Tirabosco R, Diss TC and Flanagan AM: Detection of SS18-SSX fusion transcripts in formalin-fixed paraffin-embedded neoplasms: analysis of conventional RT-PCR, qRT-PCR and dual color FISH as diagnostic tools for synovial sarcoma. Mod Pathol 20: 482-496, 2007.

9. Sun B, Sun Y, Wang J, Zhao X, Zhang S, Liu Y, Li X, Feng Y, Zhou $\mathrm{H}$ and Hao $\mathrm{X}$ : The diagnostic value of SYT-SSX detected by reverse transcription-polymerase chain reaction (RT-PCR) and fluorescence in situ hybridization (FISH) for synovial sarcoma: a review and prospective study of 255 cases. Cancer Sci 99: 1355-1361, 2008.

10. Kawauchi S, Fukuda T, Chochi Y, Kondo T, Oga A and Sasaki K: Reverse transcription-polymerase chain reaction in situ hybridization for SYT-SSX fusion gene transcripts in synovial sarcomas. Int J Mol Med 16: 763-766, 2005.

11. Pelosi G,Luzzatto F, Landoni F, Staffa N, Maggioni A, Braidotti P, Cabras A, Aiello A, Del Curto B and Viale G: Poorly differentiated synovial sarcoma of the vagina: first reported case with immunohistochemical, molecular and ultrastructural data. Histopathology 50: 808-810, 2007

12. Mitsuhashi A, Nagai Y, Suzuka K, Yamazawa K, Nojima T, Nikaido T, Ishikura H, Matsui $\mathrm{H}$ and Shozu M: Primary synovial sarcoma in fallopian tube; a case report and literature review. Int J Gynecol Pathol 26: 34-37, 2006.

13. Smith CJ, Ferrier AJ, Russell P and Danieletto S: Primary synovial sarcoma of the ovary: first reported case. Pathology 37 : 385-387, 2005.

14. Surace C, Panagopoulos I, Pålsson E, Rocchi M, Mandahl N and Mertens F: A novel FISH assay for SS18-SSX fusion type in synovial sarcoma. Lab Invest 84: 1185-1192, 2004. 